\title{
SINGULARIDAD DE SOLUCIONES DE UNA ECUACIÓN DE KIRCHHOFF NO LINEAL CON TÉRMINO DISIPATIVO
}

\section{Teófanes Quispe Méndez ${ }^{1}$}

RESUMEN.- Se estudia la singularidad en tiempo finito de las soluciones locales del problema mixto para un tipo de ecuación de Kirchhoff no lineal con término disipativo.

PALABRAS CLAVE: Singularidad de Soluciones, Solución Local, Ecuación de Kirchhoff no Lineal, Ecuación Hiperbólica no Lineal.

\section{BLOW-UP OF SOLUTIONS OF A NONLINEAR KIRCHHOFF'S EQUATION WITH DAMPING TERM}

\begin{abstract}
We study the blow-up in finite time of the local solutions to the mixed problem for a type of nonlinear Kirchhoff's equation with damping term.
\end{abstract}

KEYWORDS: Blow-up of Solutions, Nonlinear Kirchhoff's Equations, Nonlinear Hyperbolic Equations.

\section{INTRODUCCIÓN}

En este artículo consideramos el problema de valores iniciales y frontera

$$
\begin{array}{ll}
u_{t t}+\alpha(x) u_{t}-M\left(\int_{\Omega}|\nabla u|^{2} d x\right) \Delta u=f(u) & \text { en } \Omega \times[0, \infty[ \\
u=0 & \text { en } \partial \Omega \times[0, \infty[ \\
u(x, 0)=u_{0}(x), u_{t}(x, 0)=u_{1}(x) & \text { en } \Omega
\end{array}
$$

donde $\Omega$ es un conjunto abierto y acotado de $\mathbb{R}^{n}$ con frontera bien regular $\partial \Omega, \nabla$ es el gradiente, $\Delta$ el operador laplaciano, $\alpha(x)$ una función real no negativa para $x \in \Omega, M(s)$ función real positiva para $s \geq 0$ y $f(s)$ función real no lineal para $s \in \mathbb{R}$.

En caso $n=1$, el problema (1) - (3) describe las vibraciones transversales no lineales de una cuerda elástica fuertemente tensa entre dos puntos fijos $x=0$ y $x=L$ en el eje $x$ del plano $x u$. La ecuación resultante es

$$
\left.p h u_{t t}+\alpha(x) u_{t}-\left(p_{0}+\frac{E h}{2 L} \int_{0}^{L}\left|u_{x}\right|^{2} d x\right) u_{x x}=f(u) \quad \text { en } \quad\right] 0, L[\times[0, \infty[,
$$


donde $u=u(x, t)$ es el desplazamiento transversal en el espacio de coordenadas $x$ y en el tiempo $t, \rho$ denota la densidad de masa, $h$ el área de la sección transversal de la cuerda, $p_{0}$ es la tensión inicial, $E$ es el módulo de Young del material, $\alpha(x)$ se refiere al módulo de resistencia en la coordenada $x$ por intervención de la fuerza amortiguadora, y $f(u)$ la fuerza restauradora. Para el caso $\alpha \equiv f \equiv 0$, la ecuación fue propuesta primero por Kirchhoff [8], por lo que se le denomina ecuación de Kirchhoff. Para el caso general $n \geq 1$, el problema (1) - (3) tiene diversas aplicaciones, tales como por ejemplo en el área de la óptica no lineal, física del plasma, mecánica de fluidos, etc.

Diferentes autores investigaron la solubilidad del problema (1) - (3), para diferentes condiciones de las funciones $\alpha, M$ y $f$, la dimensión $n$ y los datos complementarios, entre ellos podemos mencionar a Ikehata [6], Ikehata and Okazawa [7], Maciel and Lima [10], Ono [12], Cabanillas Lapa [3], [4], Aassila and Benaissa [1].

Son escasos los resultados sobre el estudio de la singularidad en tiempo finito de las soluciones locales del problema (1) - (3) para diferentes hipótesis sobre las funciones $\alpha, M$ y $f$. Bainov and Minchev [2] investigaron la singularidad pará cada pequeño entorno del origen de coordenadas de la variable espacial cuando $\alpha(x) \equiv 0, \quad M(s) \equiv 1$ y $f(s)=g\left(|s|^{2}\right) s, \quad$ Ikehata and Okazawa [7] obtuvieron singularidad con energía inicial positiva para el caso en que $\alpha(x) \equiv 0, M(s) \equiv a+2 b s, f(s)=\mu s^{3}$ y $n=3$. Cuando $\alpha(x) \equiv 0, M(s)=a+(\rho+2) b s^{\rho+1}, f(s) \equiv \gamma|s|^{2 \rho+2} s$ y $1 \leq n \leq 3$, Carrillo Díaz [5] obtuvo singularidad con energías iniciales positiva y no positiva. Para el caso en que $\quad \alpha(x)=\delta$ constante no negativa, $M(s)=a+b s^{\gamma}, f(s)=|s|^{\rho} s$ y $n \geq 1$, Ono [12] investigó la singularidad con energía inicial positiva y no positiva.

El propósito del presente trabajo es discutir la propiedad de singularidad en tiempo finito de las soluciones locales del problema (1) - (3) con energía inicial $E(0)<0, E(0)=0$ y $E(0)>0$, cuando las funciones $\alpha, M$ y $f$ cumplan ciertas condiciones y $n \geq 1$. En la discusión emplearemos las estrategias seguidas por Ono [12] y Li and Tsai [9].

\section{PRELIMINARES}

Sea $\Omega$ un conjunto abierto y acotado de $\mathbb{R}^{n}$ con frontera bien regular $\partial \Omega$. Denotamos el producto interno y la norma de $L^{2}(\Omega)$ y $L^{\rho}(\Omega)$, con $(.,$.$) y |\cdot|_{L^{p} \Omega}$, respectivamente, para $1 \leq p \leq \infty$. Además $a(.,$.$) y \|$.$\| , denotarán el producto interno y la norma de H_{0}^{1}(\Omega)$, donde $a(u, v)=\int_{\Omega} \nabla u . \nabla v d x$ es la forma de Dirichelet.

Sea $X$ un espacio de Banach, $0<T \leq \infty$ y $1 \leq p \leq \infty$. Representamos con $L^{p}(0, T ; X)$ al espacio de Banach de las funciones vectoriales $\left.u:\right] 0, T[\rightarrow X$ tales que 
son medibles y $\|u(t)\|_{X} \in L^{p}(0, T)$, con la norma

$$
\begin{gathered}
\|u\|_{L^{p}(0, T ; X)}=\left(\int_{0}^{T}\|u(t)\|_{X}^{p} d t\right)^{\frac{1}{p}}, 1 \leq p<\infty \\
\|u\|_{L^{\infty}(0, T ; X)}=\sup _{0<t<T} \operatorname{ess}\|u(t)\|_{X}, p=\infty .
\end{gathered}
$$

Denotamos $v^{\prime}=v_{t}, v^{\prime \prime}=v_{t}$ y $v(t)(x)=v(x, t)$.

\section{Hipotesis.}

Impondremos las siguientes condiciones sobre las funciones reales $\alpha(x), M(s)$ y $f(s)$

(H1) $\alpha \in W^{1, \infty}(\Omega)$ que satisface

$$
\alpha(x) \geq 0, \forall x \in \Omega \text {. }
$$

(H2) $M \in C^{1}([0, \infty[)$ satisfaciendo

$$
\begin{gathered}
M(s) \geq m_{0}, \forall s \geq 0, \\
(2 \gamma+1) \widehat{M}(s) \geq s M(s), \forall s \geq 0,
\end{gathered}
$$

donde $m_{0}$ y $\gamma$ son constantes positivas y $\widehat{M}(s):=\int_{0}^{s} M(\xi) d \xi$.

(H3) $f \in C^{1}(\mathbb{R})$ satisfaciendo

$$
\begin{gathered}
|f(s)| \leq k_{1}|s|^{\rho+1} \text { y }\left|f^{\prime}(s)\right| \leq k_{2}|s|^{\rho}, \forall s \in \mathbb{R}, \\
f(s) s \geq 2(2 \gamma+1) F(s), \quad \forall s \in \mathbb{R},
\end{gathered}
$$

donde $k_{1}$ y $k_{2}$ son constantes positivas, $\gamma$ es la misma constante de (6),

$$
\begin{aligned}
& F(s):=\int_{0}^{s} f(\xi) d \xi, \mathrm{y} \\
& \quad 0<\rho \leq \frac{2}{n-2} \text { para } n \geq 3 \text { o } \rho>0 \text { para } 1 \leq n \leq 2 .
\end{aligned}
$$
trabajo.

Los siguientes resutados serán utilizados en la demostración de la parte principal del

Lema 2.1. (Desigualdad Generalizada de Gronwall [11]). Sea $f:[0, \infty[\rightarrow[0, \infty[$ continua, $g:] 0, \infty[\rightarrow] 0, \infty[$ continua y no decreciente y sea $C$ una constante positiva, tal que se satisface la desigualdad

$$
f(t) \leq C+\int_{0}^{t} g(f(s)) d s, \quad \forall t \in[0, \infty[.
$$

Entonces,

$$
f(t) \leq G^{-1}\left(T_{*}\right)<\infty, \quad \forall t \in\left[0, T_{*}\right],
$$


para cualquier número fijo $T_{*}<G(\infty)$, donde

$$
G(t)=\int_{C}^{t} \frac{d s}{g(s)}, \forall t \in[C, \infty[
$$

Más aún, si $G(\infty)=\infty$, entonces

$$
f(t) \leq G^{-1}(t), \quad \forall t \in[0, \infty[
$$

Definición 2.1. Una función $u: \Omega \times[0, T[\rightarrow \mathbb{R}$ es llamada solución del problema (1) (3) sobre $[0, T[$ sí

$$
\begin{aligned}
& u \in L^{\infty}\left(0, T ; H_{0}^{1}(\Omega) \cap H^{2}(\Omega)\right), \\
& u^{\prime} \in L^{\infty}\left(0, T ; H_{0}^{1}(\Omega)\right), \\
& u^{\prime \prime} \in L^{\infty}\left(0, T ; L^{2}(\Omega)\right), \\
&\left(u^{\prime \prime}(t), v\right)+\left(\alpha u^{\prime}(t), v\right)+M\left(\|u(t)\|^{2}\right) a(u(t), v)=(f(u(t)), v),
\end{aligned}
$$

$\forall v \in H_{0}^{1}(\Omega)$ en el sentido de $D^{\prime}(0, T)$.

$$
u(x, 0)=u_{0}(x) \text { y } u^{\prime}(x, 0)=u_{1}(x) .
$$

Teorema 2.1 (Solución Local). Supongamos que las funciones $\alpha, M$ y $f$ satisfacen (4), (5) y (7), respectivamente. Si $u_{0} \in H_{0}^{1}(\Omega) \cap H^{2}(\Omega)$ y $u_{1} \in H_{0}^{1}(\Omega)$, entonces existe un único intervalo $\left[0, T_{M}\left[\right.\right.$ con $0<T_{M} \leq \infty$ y una única solución del problema (1) - (3) sobre $\left[0, T_{M}[\right.$.

Demostración. El Teorema se demuestra utilizando el método de Galerkin; el método de estimativas de Tartar, en el cual se utiliza la inmersión de Sobolev con (9) y la desigualdad generalizada de Gronwall; resultados de Compacidad de Lions; y finalmente el Lema de Zorn. Asi se obtiene el resultado del teorema, ver por ejemplo Quispe Méndez [13].

Observación. Supongamos que la función $\alpha$ satisface

$$
\alpha \in L^{\infty}\left(0, \infty ; W^{1, \infty}(\Omega)\right) \quad \text { y } \quad \alpha(x, t) \geq 0, \forall(x, t) \in \Omega \times[0, \infty[.
$$

Si reemplazamos la hipótesis $(H 1)$ por (10), se obtiene los mismos resultados del Teorema 2.1 .

Lema 2.2 (Li-Tsai [9] ). Sea $\gamma>0$ y sea $b(t): \mathbb{R}^{+} \rightarrow \mathbb{R}^{+}$una función de clase $C^{2}$ que satisface

$$
b^{\prime \prime}(t)-4(\gamma+1) b^{\prime}(t)+4(\gamma+1) b(t) \geq 0, \forall t \geq 0 .
$$

Si $b^{\prime}(0)>r_{2} b(0)$, entonces $b^{\prime}(t)>0, \forall t>0$, donde 


$$
r_{2}=2(\gamma+1)-2 \sqrt{(\gamma+1) \gamma}
$$

es la menor raíz de la ecuación cuadrática $r^{2}-4(\gamma+1) r+4(\gamma+1)=0$.

Lema 2.3 (Li-Tsai [9] ). Sea $\gamma>0$. Si $J(t)$ es una función no creciente en $\left[t_{0}, \infty\left[, t_{0} \geq 0\right.\right.$ y satisface la inecuación diferencial

$$
\left[J^{\prime}(t)\right]^{2} \geq a+b[J(t)]^{2+\frac{1}{\gamma}}, \text { para } t \geq t_{0}
$$

donde $a>0 \quad y \quad b \in \mathbb{R}$, entonces existe un número real positivo $T_{*}$ tal que $\lim _{t \rightarrow T_{*}^{-}} J(t)=0_{y}$ una cota superior de $T_{*}$ puede ser estimado, respectivamente, en los siguientes casos:

(i) cuando $b<0$ y $J\left(t_{0}\right)<\min \left\{1, \sqrt{\frac{a}{-b}}\right\}$,

$$
T_{*} \leq t_{0}+\frac{1}{\sqrt{-b}} \ln \left(\frac{\sqrt{\frac{a}{-b}}}{\sqrt{\frac{a}{-b}}-J\left(t_{0}\right)}\right) \text {, }
$$

(ii) cuando $b=0$,

$$
T_{*} \leq t_{0}+\frac{J\left(t_{0}\right)}{\sqrt{a}}
$$

(iii) cuando $b>0$,

$$
\begin{aligned}
& \qquad T_{*} \leq t_{0}+2^{\frac{3 \gamma+1}{2 \gamma}} \frac{\gamma c}{\sqrt{a}}\left(1-\left[1+c J\left(t_{0}\right)\right]^{-\frac{1}{2 \gamma}}\right), \\
& \text { donde } c=\left(\frac{a}{b}\right)^{2+\frac{1}{\gamma}} .
\end{aligned}
$$

\section{EL RESULTADO PRINCIPAL}

El objetivo central del presente trabajo es discutir la propiedad de singularidad en tiempo finito de las soluciones del problema (1) - (3) sobre un intervalo maximal $\left[0, T_{M}[\right.$, es decir que una solución $u$ del problema (1) - (3) sobre $\left[0, T_{M}[\right.$ tiene la propiedad

$$
T_{M}<\infty \quad y \quad \lim _{t \rightarrow T_{M}^{-}}|u(t)|_{L^{2}(\Omega)}=\infty
$$


Definición 3.1. La función energía $E(t)$ del problema (1) - (3), se define en el espacio $H_{0}^{1}(\Omega)$ por

$$
E(t):=\frac{1}{2}\left|u^{\prime}(t)\right|_{L^{2}(\Omega)}^{2}+\frac{1}{2} \widehat{M}\left(\|u(t)\|^{2}\right)-\int_{\Omega} F(u(x, t)) d x, \quad t \geq 0,
$$

donde

$$
\widehat{M}(s):=\int_{0}^{s} M(\xi) d \xi \quad y \quad F(s):=\int_{0}^{s} f(\xi) d \xi .
$$

Lema 3.1. Si u es una solución del problema (1) - (3) sobre $\left[0, T_{M}[\right.$, entonces,

$$
E(t)+\int_{0}^{t}\left|\sqrt{\alpha} u^{\prime}(\tau)\right|_{L^{2}(\Omega)}^{2} d \tau=E(0), \quad 0 \leq t<T_{M},
$$

donde $E(0)$ es la energía inicial definida por

$$
E(0):=\frac{1}{2}\left|u_{1}\right|_{L^{2}(\Omega)}^{2}+\frac{1}{2} \widehat{M}\left(\left\|u_{0}\right\|^{2}\right)-\int_{\Omega} F\left(u_{0}(x)\right) d x
$$

Demostración. Multiplicando la ecuación (1) por $u_{t}$ integrando sobre $\Omega$ y aplicando el teorema de la divergencia, obtenemos $E^{\prime}(t)=0$. De aqui, se obtiene el resultado.

Lema 3.2. Si u es una solución del problema (1) - (3) sobre $\left[0, T_{M}[\right.$, entonces,

$$
\begin{aligned}
2\left[\left|u^{\prime}(t)\right|_{L^{2}(\Omega)}^{2}\right. & \left.+\int_{0}^{t}\left|\sqrt{\alpha} u^{\prime}(\tau)\right|_{L^{2}(\Omega)}^{2} d \tau\right]=\frac{1}{2} A^{\prime \prime}(t)+2 E(0) \\
& +M\left(\|u(t)\|^{2}\right)\|u(t)\|^{2}-\widehat{M}\left(\|u(t)\|^{2}\right) \\
& +2 \int_{\Omega} F(u(x, t)) d x-(f(u(t)), u(t)), \quad 0 \leq t<T_{M}, \\
M\left(\|u(t)\|^{2}\right)\|u(t)\|^{2}+\widehat{M}\left(\|u(t)\|^{2}\right)=2 E(0)-\frac{1}{2} A^{\prime \prime}(t) & +2 \int_{\Omega} F(u(x, t)) d x+(f(u(t)), u(t)) \\
& -2 \int_{0}^{t}\left|\sqrt{\alpha} u^{\prime}(\tau)\right|_{L^{2}(\Omega)}^{2} d \tau, \quad 0 \leq t<T_{M},
\end{aligned}
$$

donde

$$
A(t):=|u(t)|_{L^{2}(\Omega)}^{2}+\int_{0}^{t}\left|\sqrt{\alpha} u^{\prime}(\tau)\right|_{L^{2}(\Omega)}^{2} d \tau+\left(T_{0}-t\right)\left|\sqrt{\alpha} u_{0}\right|^{2} \geq 0,
$$

y $T_{0}$ es una constante positiva que será determinado más adelante. 
Demostración. Derivando (14) una y dos veces, obtenemos

$$
A^{\prime}(t)=2\left[\left(u^{\prime}(t), u(t)\right)+\int_{0}^{t}\left(\alpha u^{\prime}(\tau), u(\tau)\right) d \tau\right]
$$

y

$$
A^{\prime \prime}(t)=2\left[\left|u^{\prime}(t)\right|_{L^{2}(\Omega)}^{2}-M\left(\|u(t)\|^{2}\right)\|u(t)\|^{2}+(f(u(t)), u(t))\right] .
$$

De (11) y (16), se obtiene (12). También de (11) y (16) resultan, respectivamente,

$$
\begin{aligned}
\widehat{M}\left(\|u(t)\|^{2}\right)=2 E(0) & +2 \int_{\Omega} F(u,(x, t)) d x \\
& -2 \int_{0}^{t}\left|\sqrt{\alpha} u^{\prime}(\tau)\right|_{L^{2}(\Omega)}^{2} d \tau-\left|u^{\prime}(t)\right|_{L^{2}(\Omega)}^{2}
\end{aligned}
$$

y

$$
M\left(\|u(t)\|^{2}\right)\|u(t)\|^{2}=\left|u^{\prime}(t)\right|_{L^{2}(\Omega)}^{2}+(f(u(t)), u(t))-\frac{1}{2} A^{\prime \prime}(t) .
$$

Sumando estos dos resultados, se obtiene (13).

Lema 3.3. Supongamos que las funciones $\alpha, M$ y $f$ satisfacen (4), (6) y (8). Si u es una solución del problema (1) - (3) sobre $\left[0, T_{M}[\right.$ y uno de los siguientes casos se satisface:

(i) $E(0)<0$,

(ii) $E(0)=0 \quad y \quad A^{\prime}(0)>0$,

(iii) $E(0)>0 \quad y \quad A^{\prime}(0)>[2(\gamma+1)-2 \sqrt{(\gamma+1) \gamma}]\left[A(0)+\frac{(1+2 \gamma) E(0)}{1+\gamma}\right]$,

donde

$$
A(0):=\left|u_{0}\right|_{L^{2}(\Omega)}^{2}+T_{0}\left|\sqrt{\alpha} u_{0}\right|^{2} \quad y \quad A^{\prime}(0):=2\left(u_{1}, u_{0}\right)
$$

entonces

$$
\left.A^{\prime}(t)>0, \forall t \in\right] t_{0}, T_{M}[
$$

donde $t_{0}:=\max \left\{\frac{A^{\prime}(0)}{4(1+2 \gamma) E(0)}, 0\right\}$ en el caso (i) y $t_{0}:=0$ en los casos (ii) e (iii).

Demostración. Para $\gamma>0$, definamos la función

$$
J(t):=[A(t)]^{-\gamma}, \quad \forall t \in\left[0, T_{M}[\right.
$$

Donde $A(t)$ está definida por (14). Derivando (18) una y dos veces, obtenemos 


$$
J^{\prime}(t)=-\gamma[A(t)]^{-(\gamma+1)} A^{\prime}(t)
$$

y

$$
J^{\prime \prime}(t)=\gamma[A(t)]^{-(\gamma+2)}\left[(\gamma+1)\left[A^{\prime}(t)\right]^{2}-A(t) A^{\prime \prime}(t)\right]
$$

De (15) por la desigualdad triangular y la desigualdad de Cauchy-Schwarz, se obtiene

$$
\left[A^{\prime}(t)\right]^{2} \leq 4 A(t)\left[\left|u^{\prime}(t)\right|_{L^{2}(\Omega)}^{2}+\int_{0}^{t}\left|\sqrt{\alpha} u^{\prime}(\tau)\right|_{L^{2}(\Omega)}^{2} d \tau\right]
$$

De (20) y (21), se tiene

$$
J^{\prime \prime}(t) \leq-\gamma[A(t)]^{-(\gamma+1)} K(t)
$$

Donde

$$
K(t):=A^{\prime \prime}(t)-4(\gamma+1)\left[\left|u^{\prime}(t)\right|_{L^{2}(\Omega)}^{2}+\int_{0}^{t}\left|\sqrt{\alpha} u^{\prime}(\tau)\right|_{L^{2}(\Omega)}^{2} d \tau\right]
$$

Por (12) y (13), obtenemos

$$
\begin{aligned}
K(t)= & -4(2 \gamma+1) E(0)+2\left[(2 \gamma+1) \widehat{M}\left(\|u(t)\|^{2}\right)\right. \\
& \left.-M\left(\|u(t)\|^{2}\right)\|u(t)\|^{2}\right]+2[(f(u(t), u(t)) \\
& \left.-2(2 \gamma+1) \int_{\Omega} F(u(x, t)) d x\right]+4 \gamma \int_{0}^{t}\left|\sqrt{\alpha} u^{\prime}(\tau)\right|_{L^{2}(\Omega)}^{2} d \tau
\end{aligned}
$$

Por (6) y (8), de (24), resulta

$$
\begin{gathered}
A^{\prime \prime}(t)-4(\gamma+1)\left[\left|u^{\prime}(t)\right|_{L^{2}(\Omega)}^{2}+\int_{0}^{t}\left|\sqrt{\alpha} u^{\prime}(\tau)\right|_{L^{2}(\Omega)}^{2} d \tau\right] \\
\geq-4(2 \gamma+1) E(0)
\end{gathered}
$$

Ahora vamos a considerar tres casos diferentes de acuerdo al signo de la energía inicial $E(0)$.

(i) Si $E(0)<0$, por integración de $(25)$, resulta

$$
A^{\prime}(t) \geq A^{\prime}(0)-4(2 \gamma+1) E(0) t, \text { para } t \geq 0
$$

Asi tenemos $A^{\prime}(t)>0$, para $t>t_{0}$, donde 


$$
t_{0}:=\max \left\{\frac{A^{\prime}(0)}{4(1+2 \gamma) E(0)}, 0\right\}
$$

(ii) Si $E(0)=0$ y $A^{\prime}(0)>0$, entonces $A^{\prime \prime}(t) \geq 0$, para $t \geq 0$. Integrando, resulta $A^{\prime}(t)>0$, para $t>0$.

(iii) Si $E(0)>0 \quad$ y $A^{\prime}(0)>r_{2}\left[A(0)+\frac{(2 \gamma+1) E(0)}{\gamma+1}\right]$, donde $r_{2}:=2(\gamma+1)-2 \sqrt{(\gamma+1) \gamma}$.

De (15), por la desigualdad triangular y la desigualdad de Cauchy - Schwarz, resulta

$$
A^{\prime}(t) \leq A(t)+\left|u^{\prime}(t)\right|_{L^{2}(\Omega)}^{2}+\int_{0}^{t}\left|\sqrt{\alpha} u^{\prime}(\tau)\right|_{L^{2}(\Omega)}^{2} d \tau
$$

De (25) y (26), se tiene

$$
A^{\prime \prime}(t)-4(\gamma+1) A^{\prime}(t)+4(\gamma+1) A(t)+4(2 \gamma+1) E(0) \geq 0 .
$$

Definimos la función

$$
b(t):=A(t)+\frac{(1+2 \gamma) E(0)}{1+\gamma}, \text { para } t \geq 0 .
$$

Entonces $b(t)$ satisface las condiciones del Lema 2.2. Así se tiene $A^{\prime}(t)>0$, para $t>0$.

Teorema 3.1 (Singularidad de Soluciones). Supongamos que las funciones $\alpha, M$ y $f$ satisfacen las hipótesis (H1), (H2) y (H3), respectivamente. Si u es una solución del problema (1) - (3) sobre $\left[0, T_{M}\left[\right.\right.$ con datos iniciales $u_{0} \in H_{0}^{1}(\Omega) \cap H^{2}(\Omega)$ y $u_{1} \in H_{0}^{1}(\Omega)$ satisfaciendo uno de los siguientes casos:

(i) $E(0)<0$,

(ii) $E(0)=0 \quad y \quad A^{\prime}(0)>0$,

(iii) $\frac{\left[A^{\prime}(0)\right]^{2}}{8 A(0)}>E(0)>0 \quad y \quad A^{\prime}(0)>r_{2}\left[A(0)+\frac{(1+2 \gamma) E(0)}{\gamma+1}\right]$,

entonces $T_{M}<\infty$ y $\lim _{t \rightarrow T_{M}^{-}} A(t)=\infty$. Además, el tiempo finito $T_{M}$ es estimado, en el caso $(i)$,

$$
T_{M} \leq t_{0}-\frac{J\left(t_{0}\right)}{J^{\prime}\left(t_{0}\right)}
$$

Además, si $J\left(t_{0}\right) \leq \min \left\{1, \sqrt{\frac{a}{-b}}\right\}$, entonces

$$
T_{M} \leq t_{0}+\frac{1}{\sqrt{-b}} \ln \left(\frac{\sqrt{\frac{a}{-b}}}{\sqrt{\frac{a}{-b}}-J\left(t_{0}\right)}\right) .
$$


En el caso (ii),

$$
T_{M} \leq t_{0}-\frac{J\left(t_{0}\right)}{J^{\prime}\left(t_{0}\right)}
$$

En el caso (iii),

$$
T_{M} \leq t_{0}+2^{\frac{3 \gamma+1}{2 \gamma}} \frac{\gamma c}{\sqrt{a}}\left(1-\left[1+c J\left(t_{0}\right)\right]^{-\frac{1}{2 \gamma}}\right)
$$

Aqui $c:=\left(\frac{a}{b}\right)^{2+\frac{1}{\gamma}}$ con $a:=\gamma^{2}\left[A\left(t_{0}\right)\right]^{-2(\gamma+1)}\left(\left[A^{\prime}\left(t_{0}\right)\right]^{2}-8 E(0) A\left(t_{0}\right)\right) y b:=8 \gamma^{2} E(0)$.

En el caso $(i), t_{0}:=\max \left\{\frac{A^{\prime}(0)}{4(1+2 \gamma) \mathrm{E}(0)}, 0\right\}$ y $t_{0}:=0$ en los casos (ii) y (iii).

Demostración. De (22) y (25), resulta

$$
J^{\prime \prime}(t) \leq 4 \gamma(2 \gamma+1) E(0)[A(t)]^{-(\gamma+1)}, \text { para } t \geq t_{0} .
$$

Por (17) y (19), se tiene

$$
J^{\prime}(t)<0 \text {, para } t>t_{0}
$$

Multiplicando (31) por $J^{\prime}(t)$ y luego integrando de $t_{0} a t$, se obtiene

$$
\left[J^{\prime}(t)\right]^{2} \geq a+b[J(t)]^{2+\frac{1}{\gamma}}, \text { para } t>t_{0}
$$

donde

$$
\begin{aligned}
a: & =\left[J^{\prime}(t)\right]^{2}-8 \gamma^{2} E(0)\left[J\left(t_{0}\right)\right]^{2+\frac{1}{\gamma}} \\
& =\gamma^{2}\left[A\left(t_{0}\right)\right]^{-2(\gamma+1)}\left(\left[A^{\prime}\left(t_{0}\right)\right]^{2}-8 E(0) A\left(t_{0}\right)\right)
\end{aligned}
$$

y

$$
b:=8 \gamma^{2} E(0)
$$

Observemos que $a>0$ si y sólo si $E(0)<\frac{\left[A^{\prime}\left(t_{0}\right)\right]^{2}}{8 A(0)}$.

En el caso que $E(0)<0$, por (22) y $(25)$, se obtiene la singularidad y la estimativa del tiempo finito $T_{M} \leq t_{0}-\frac{J\left(t_{0}\right)}{J^{\prime}\left(t_{0}\right)}$. Para los demás casos, por (32) y Lema 2.3, se obtiene los resultados del teorema. 
Para terminar la prueba, debemos elegir la constante positiva $T_{0}$ de (14) tal que satisfaga (27),

$$
t_{0}-\frac{J\left(t_{0}\right)}{J^{\prime}\left(t_{0}\right)} \leq T_{0} .
$$

Para (28),

$$
J\left(t_{0}\right) \leq \min \left\{1, \sqrt{\frac{a}{-b}}\right\} \text { y } t_{0}+\frac{1}{\sqrt{-b}} \ln \left(\frac{\sqrt{\frac{a}{-b}}}{\sqrt{\frac{a}{-b}}-J\left(t_{0}\right)}\right) \leq T_{0}
$$

Para (29),

$$
t_{0}-\frac{J\left(t_{0}\right)}{J^{\prime}\left(t_{0}\right)} \leq T_{0}
$$

Para (30),

$$
t_{0}+2^{\frac{3 \gamma+1}{2 \gamma}} \frac{\gamma c}{\sqrt{a}}\left(1-\left[1+c J\left(t_{0}\right)\right]^{-\frac{1}{2 \gamma}}\right) \leq T_{0} .
$$

Finalmente, se concluye la demostración.

Comentario. La aplicación del Lema 2.3, permite estudiar la singularidad en tiempo finito de las soluciones, de una variedad de ecuaciones diferenciales de evolución.

En el futuro, sería interesante estudiar la singularidad en tiempo finito de las soluciones, del problema mixto asociado a la ecuación diferencial no lineal

$$
u_{t t}+\alpha(x, t) g\left(u_{t}\right)-M\left(\int_{\Omega}|\nabla u|^{2} d x\right) \Delta u=f(u) \quad \text { en } \quad \Omega \times[0, \infty[\text {, }
$$

donde $\alpha(x, t)$ es una función real no negativa para $(x, t) \in \Omega \times[0, \infty[, g(s)$ y $f(s)$ funciones reales que satisfacen ciertas condiciones para $s \in \mathbb{R}$, y $M(s)$ función real positiva para $s \geq 0$.

En conclusión el Teorema 3.1, en cierta manera generaliza los resultados mencionados en la introducción, aunque las estimativas del tiempo finito $T_{M}$ no son las óptimas. 
Quispe: Singularidad de Soluciones de una Ecuación de Kirchhoff ...

\section{REFERENCIA BIBLIOGRÁFICAS}

[1] Aassila, M. and Benaissa, A., Existence of Global Solutions to a Quasilinear Wave Equation with General Nonlinear Damping, Electronic Journal of Differential Equations, Vol. 2002, No 91, 1-22 (2002).

[2] Bainov, D. D. and Minchev, E., Nonexistence of Global Solutions of the Initial-Boundary Value Problem for the Nonlinear Klen-Gordon Equation, J. Math. Phys., Vol. 36, $\mathrm{N}^{\mathrm{o}} 2$, 756-762 (1995).

[3] Cabanillas Lapa, E., Sobre una Ecuación de Kirchhoff no lineal con Término Disipativo, sin Datos en el Pozo de Potencial, Atas do $48^{\circ}$ SBA, 737-742 (1988).

[4] Cabanillas Lapa, E. Existencia Global y Comportamiento Asintótico para una Ecuación de Kirchhoff con Término Disipativo de Coeficiente Variable, PESQUIMAT, Vol. III, N ${ }^{\circ}$ 2, 44-50 (2000).

[5] Carrillo Díaz, L. E., On Blow-Up for the Nonlinear Kirchhoff Equations, Atas do $42^{\circ}$ SBA, 513-522 (1995).

[6] Ikehata, R., On Solutions to Some Quasilinear Hyperbolic Equations with Nonlinear Inhomogeneous Terms, Nonlinear Analysis, T.M.A., Vol. 17, N² 181-201 (1991).

[7] Ikehata, R. and Okazawa, N., A Class of Second Order Quasilinear Evolution Equations, Journal of Differential Equations 114, 106-131 (1994).

[8] Kirchhoff, G., Vorlesungen über Mechanik, Tauber Leipzig (1883).

[9] Li, M. R. and Tsai, L. Y., On a System of Nonlinear Wave Equations, Taiwanese Journal of Mathematics, Vol. 7, No 4, 557-573 (2003).

[10] Maciel, A. and Lima, D.A., Nonlinear Perturbation of Kirchhoff-Carrier's Equations, Atas do $42^{\circ}$ SBA, 293-300 (1995).

[11] Nishihara, K., On a Global Solution of Some Quasilinear Hyperbolic Equation, Tokio J. Math, Vol. 7, N 2, 437-459 (1984).

[12] Ono, K., Global Existence, Decay, and Blow-up of Solutions for some Mildly Degenerate Nonlinear Kirchhoff Strings, Journal of Differential Equations 137, 273-301 (1997).

[13] Quispe Méndez, T., Singularidad en Tiempo Finito para un Sistema de Kirchhoff, Tesis de Maestría, UNMSM (1998). 